Paper published in:

D. Gallipoli, A.W. Bruno (2017).

A bounding surface compression model with a unified virgin line for saturated and unsaturated soils.

Géotechnique, 67(8): 703-712

http://dx.doi.org/10.1680/jgeot.16.P.145

\title{
A BOUNDING SURFACE COMPRESSION MODEL WITH A UNIFIED VIRGIN LINE FOR SATURATED AND UNSATURATED SOILS
}

\author{
Domenico Gallipoli ${ }^{1}$, Agostino Walter Bruno²
}

1 Professor, Laboratoire SIAME, Université de Pau et des Pays de l'Adour, Anglet, France, email: domenico.gallipoli@univ-pau.fr

2 Research Assistant, Laboratoire SIAME, Université de Pau et des Pays de l'Adour, Anglet, France, email: agostinowalter.bruno@univ-pau.fr

DATE OF SUBMISSION: 18/12/2016

NUMBER OF WORDS: 5956

NUMBER OF TABLES: 2

NUMBER OF FIGURES: 7

CORRESPONDING AUTHOR: Prof Domenico Gallipoli

Université de Pau et des Pays de l'Adour

Laboratoire SIAME - Bâtiment ISABTP

Allée du Parc Montaury

64600 Anglet

France

e-mail: domenico.gallipoli@univ-pau.fr 
ABSTRACT: The paper presents a bounding surface model that describes the gradual yielding of unsaturated soils subjected to isotropic loads. The model originates from consideration of the capillary bonding between soil grains, which leads to the definition of a "unified normal compression line" that is valid in both saturated and unsaturated conditions. This line has the same slope and intercept of the saturated normal compression line but is formulated in terms of a "scaled stress" variable, which takes into account the mechanical effect of capillarity by factoring the average skeleton stress (also known as Bishop's stress) with a power function of degree of saturation. The normal compression behaviour of unsaturated soils is therefore described by only one additional parameter, which is the exponent of the degree of saturation in the scaled stress expression. For over-consolidated soils, the occurrence of gradual yielding is introduced by assuming that, as the soil state moves towards the unified normal compression line, the slope of the loading curve tends towards the slope of the unified normal compression line according to an expression requiring only one extra parameter. Interestingly, this expression can be integrated in a closed form to provide a general equation for all loading paths in saturated and unsaturated conditions. Different loading curves are simply distinguished by the different values of the integration constants. Unloading paths are also simulated in a similar way. The proposed model requires a total of five parameters, which include the three standard parameters for saturated soils (i.e. the slope and intercept of the saturated normal compression line and the slope of the swelling line) plus one parameter to describe unsaturated behaviour and one parameter to describe the gradual yielding of over-consolidated soils subjected to loading.

KEYWORDS: plasticity, partial saturation, suction, constitutive relations, collapsed settlement, clays, normal consolidation, virgin loading, unsaturated soils, bounding surface 


\section{List of notations}

$C_{l} \quad$ constant of integration of loading paths

$C_{u} \quad$ constant of integration of unloading paths

$\gamma \quad$ gradual yielding parameter

$\delta_{i j} \quad$ Kronecker's delta

e void ratio

$e_{o} \quad$ boundary condition of void ratio

$e_{S} \quad$ saturated void ratio

$\kappa \quad$ slope of swelling line

$\lambda_{p} \quad$ slope of unified normal compression line

$\lambda_{r} \quad$ capillary bonding parameter

$p \quad$ mean total stress

$p^{\prime} \quad$ mean average skeleton stress or mean Bishop's stress

$p^{\text {net }} \quad$ mean net stress

$\bar{p} \quad$ mean scaled stress

$\bar{p}_{i} \quad$ image value of mean scaled stress

$\bar{p}_{o} \quad$ boundary condition of mean scaled stress

$p_{\text {ref }}^{\prime} \quad$ reference mean average skeleton stress

$\bar{p}_{\text {ref }} \quad$ reference mean scaled stress

$\sigma_{i j} \quad$ total stress tensor

$\sigma_{i j}^{\prime} \quad$ average skeleton stress tensor or Bishop's stress tensor

$\sigma_{i j}^{n e t}$ net stress tensor

$S \quad$ suction

$\bar{S} \quad$ scaled suction

$S_{r} \quad$ degree of saturation

$u_{a} \quad$ pore air pressure

$u_{w} \quad$ pore water pressure 


\section{Introduction}

Plastic models describing the mechanical behaviour of unsaturated soils have been developed over the past thirty years starting from the seminal work of Alonso et al. (1990) and the subsequent contributions of Wheeler and Sivakumar (1995) and Cui and Delage (1996). These early models have been formulated in terms of one tensorial stress variable, i.e. the net stress $\sigma_{i j}^{n e t}=\sigma_{i j}-\delta_{i j} u_{a}$, and one scalar stress variable, i.e. the suction $s=u_{a}-u_{w}\left(\sigma_{i j}\right.$ is the total stress, $\delta_{i j}$ is Kronecker's delta, $u_{a}$ is the pore air pressure and $u_{w}$ is the pore water pressure). Building upon these contributions, a number of authors have subsequently proposed alternative plastic frameworks where the net stress has been replaced by the average skeleton stress (e.g. Nuth and Laloui, 2007; Tarantino and Tombolato, 2005; Wheeler et al., 2003) or by a modified form of it (e.g. Khalili et al., 2008; Mašín and Khalili, 2008; Pereira et al., 2005). The average skeleton stress, also known as Bishop's stress, is defined as $\sigma_{i j}^{\prime}=\sigma_{i j}-\delta_{i j} u_{a}+\delta_{i j} S_{r}\left(u_{a}-u_{w}\right)=\sigma_{i j}^{\text {net }}+\delta_{i j} S_{r} s$ and has the advantage of naturally reducing to Terzaghi's effective stress in saturated conditions when the degree of saturation $S_{r}$ becomes equal to one. In some models, suction has also been replaced by an alternative scalar variable that depends on degree of saturation to improve the description of soil behaviour and to facilitate a seamless transition between saturated and unsaturated states (e.g. Hu et al., 2014; Toll and Ong, 2003; Gallipoli et al., 2003; Jommi and Di Prisco, 1994). Some authors have also considered the thermodynamic consistency of unsaturated soil behaviour and have proposed constitutive models where the tensorial and scalar stresses are work-conjugate of the corresponding strain variables (e.g. Sheng et al., 2008; Wheeler et al., 2003; Vaunat et al., 2000).

In recent years, there has been a progressive move away from classic elasto-plasticity towards alternative modelling approaches including generalized plasticity (e.g. Manzanal et al., 2011; Bolzon et al., 1996), bounding surface plasticity (e.g. Zhou et al., 2015; Khalili et al., 2008) and hypoplasticity (e.g. Mašín and Khalili, 2008). These approaches can account for additional features of plastic behaviour such as the occurrence of hysteresis during cyclic loading and the gradual yielding of the soil towards normally consolidated states. The increasing level of sophistication of unsaturated soil models has however entailed a multiplication of parameters and/or an increase in complexity of parameter calibration. 
This paper presents a new model that predicts the gradual yielding (i.e. the gradual development of irreversible plastic deformation) of both saturated and unsaturated soils under isotropic stress states by using only five parameters, which have a clear physical interpretation and are, therefore, relatively easy to calibrate. Three of the five parameters are those conventionally used to describe the isotropic behaviour of saturated soils, i.e. the slope and intercept of the saturated normal compression line and the slope of the swelling line. The extra two parameters govern instead the unsaturated behaviour and the gradual yielding of the soil, respectively.

One of the main achievements of the proposed model is the regularization of the virgin behaviour of both saturated and unsaturated soil onto a single normal compression line formulated in terms of a "scaled stress" variable, whose definition originates from a physical interpretation of the mechanism of capillary bonding. The scaled stress is defined as the average skeleton stress scaled by a power function of degree of saturation and it reduces to Terzaghi's effective stress under saturated conditions. The effect of suction and/or degree of saturation on virgin behaviour is therefore incorporated inside the definition of the scaled stress, which means that it is no longer necessary to introduce an explicit dependency of the normal compression line on any of these two variables.

\section{Capillary bonding function for normally consolidated soils}

In unsaturated soils, the presence of water menisci around particle contacts generates an inter-particle pull which increases friction at the point of contact and opposes relative slippage during virgin loading. This capillary pull is the result of both the surface tension and the suction acting inside water menisci (Lourenco et al., 2012) and is the reason why unsaturated soils can attain higher values of void ratio compared to saturated soils under the same average skeleton stress.

The stabilizing effect of water menisci can be quantified by a "capillary bonding function" linking the ratio $e / e_{s}$, between the unsaturated void ratio $e$ and the saturated void ratio $e_{s}$ at the same average skeleton stress, to a "capillary bonding variable". This capillary bonding variable provides a measure of the stabilizing effect of the inter-particle pull generated by water menisci and increases with: a) the formation of new 
menisci at particle contacts and b) the intensification of the bonding action by existing menisci. Gallipoli et al. (2003) suggested an expression for the capillary bonding variable that increases as degree of saturation reduces (leading to the formation of new water menisci) and as suction increases (leading to the intensification of the bonding action by existing menisci).

Triaxial tests have however demonstrated that, within the suction range typically covered by laboratory investigation, capillary bonding is more sensitive to changes of degree of saturation than suction, i.e. it is more sensitive to the number of menisci per unit volume of solids than to the intensity of the menisci pull. This is also consistent with the simple capillary model of Fisher (1926) who showed that, when suction increases from zero to infinity, the inter-particle pull produced by a single water meniscus located around the contact of two idealized spherical particles increases by only $50 \%$.

In agreement with the above evidence, we assume a simple expression for the capillary bonding variable which depends exclusively on degree of saturation. This variable is defined as the reciprocal of degree of saturation, i.e. $1 / S_{r}$, which means that it is equal to one under saturated conditions $\left(S_{r}=1\right)$ when no bonding menisci are present, but becomes greater than one as desaturation progresses $\left(S_{r}<1\right)$ and the number of bonding menisci increases.

Therefore, the value of the capillary bonding variable tends to infinity as the soil dries out and the degree of saturation tends to zero. In reality, however, the bonding effect of water menisci is expected to attain a maximum during desaturation when all bulk pore water disappears and the number of water menisci attains a peak. If saturation reduces further below this point, water menisci will start to vanish and the bonding variable begins to reduce back towards a value of one corresponding to the case of a completely dry soil. This is because a completely dry soil is conceptually no different from a saturated soil as, in both cases, no water menisci are present.

The above observation suggests that the proposed framework may not be adequate to describe the behaviour of very dry soils for which the bonding variable tends to infinity instead of converging back to one. Yet, the analysis of experimental data in the next sections has confirmed the validity of the formulation for degrees of saturation as low as 0.6 . This is the lowest degree of saturation available for the 
data considered in this study, which means that the model might remain valid for saturation levels even lower than 0.6.

The capillary bonding function $e / e_{s}$ is next expressed as a power function of the bonding variable $1 / S_{r}$ :

$$
\frac{e}{e_{s}}=\left(\frac{1}{S_{r}}\right)^{\lambda_{r}}
$$

where the exponent $\lambda_{r}$ is a model parameter greater than zero. When degree saturation is equal to one, Equation (1) correctly predicts that the void ratio $e$ coincides with the saturated value $e_{s}$. Conversely, when degree of saturation reduces below one, Equation (1) predicts that the void ratio $e$ is bigger than the saturated value $e_{s}$ at the same average skeleton stress, consistent with the stabilizing action of water menisci.

By taking logarithms, Equation (1) can be rewritten in the following alternative form:

$$
\log \frac{e}{e_{s}}=-\lambda_{r} \log S_{r}
$$

which suggests that, if the ratio $e / e_{s}$ is plotted against degree of saturation $S_{r}$ in a double-logarithmic scale, the parameter $\lambda_{r}$ can be simply determined as the slope of the resulting line. In this work, we make use of base 10 logarithms but the proposed formulation remains valid regardless of the chosen logarithm base.

In spite of requiring only one model parameter $\lambda_{r}$, Equation (2) is capable of accurately capturing the volumetric behavior of unsaturated soils during normal compression. This is shown in Figure 1, which presents a double-logarithmic plot of the experimental values of $e / e_{S}$, at different levels of the mean average skeleton stress $p^{\prime}=p-u_{a}+S_{r}\left(u_{a}-u_{w}\right)=p^{\text {net }}+S_{r} s$, against the corresponding values of degree of saturation $S_{r}$. This double-logarithmic plot is presented for three different sets of isotropic virgin compression data corresponding to compacted kaolin (Raveendiraraj, 2009), compacted bentonite/kaolin (Sharma, 1998) and compacted kaolin (Sivakumar, 1993), respectively. The corresponding values of the slope $\lambda_{r}$ are also reported in Figure 1. 
In Figure 1, only post-yield experimental values of the unsaturated void ratio have been used at the numerator of the fraction $e / e_{s}$ while the values of saturated void ratio at the denominator have been calculated from the analytical expression of the saturated normal compression line (see next section) at the same mean average skeleton stress. The post-yield experimental values of the unsaturated void ratio have been obtained from isotropic loading tests at constant suctions of $100 \mathrm{kPa}, 200 \mathrm{kPa}$ and $300 \mathrm{kPa}$ in the case of Sharma (1998) and Sivakumar (1993), and at constant suctions of $50 \mathrm{kPa}, 150 \mathrm{kPa}$ and $350 \mathrm{kPa}$ in the case of Raveendiraraj (2009).

\section{Unified normal compression line for saturated and unsaturated soils}

Regardless of whether a soil is saturated or unsaturated, the logarithm of void ratio during virgin compression can be determined from Equation (2) as:

$$
\log e=\log e_{s}-\lambda_{r} \log S_{r}
$$

On the right hand side of Equation (3), the logarithm of saturated void ratio is calculated, at any value of average skeleton stresses, by using the following double-logarithmic expression of the saturated normal compression line:

$$
\log e_{s}=-\lambda_{p} \log \frac{p^{\prime}}{p_{r e f}^{\prime}}
$$

where $p^{\prime}$ is the mean average skeleton stress, $p_{r e f}^{\prime}$ is the reference mean average skeleton stress at a void ratio of one and $\lambda_{p}$ is the slope of the saturated normal compression line. Recall that, in saturated conditions, the average skeleton stress is the same as Terzaghi's effective stress.

Although the normally consolidated behaviour of saturated soils is conventionally represented by a semilogarithmic line in the $e_{S}-\log p^{\prime}$ plane, a double-logarithmic expression, such as that of Equation (4), has been advocated by McDowell (2005) on theoretical grounds and by Pestana and Whittle (1995) on experimental grounds. A double-logarithmic line has also been suggested by Butterfield (1979), albeit in terms of specific volume rather than void ratio. An important advantage of using a double-logarithmic 
expression, such as that of Equation (4), is that the void ratio tends to zero as the mean average skeleton stress tends to infinity. This means that the void ratio remains always positive regardless of the stress level. This is not true for a semi-logarithmic expression, which would incorrectly predict negative values of void ratio as the mean average skeleton stress tends to infinity.

Figure 2 demonstrates the good fit of the normal compression line of Equation (4) to the saturated loading data of the three soils considered in this work. Figure 2 also shows the values of the slope $\lambda_{p}$ and intercept $p_{\text {ref }}^{\prime}$ of each normal compression line. The initial data points, at small values of $p^{\prime}$, correspond to overconsolidated states and therefore deviate from the interpolating line. Because of this, these data points have not been included in the fit of Equation (4). Inspection of Figure 2 supports the assumption of a double-logarithmic expression for the saturated normal compression line of the three soils considered in this study, though it is not possible to guarantee that the same will apply to all soils.

Note that Raveendiraraj (2009) reported higher values of the compaction void ratio for samples tested in saturated conditions compared to samples tested in unsaturated conditions. This was an unexpected result because all samples were ostensibly compacted in the same way regardless of the testing path to which they were subjected after compaction (i.e. the discrepancy refers to the values of void ratio measured after compaction and not after saturation or suction equalization). Raveendiraraj (2009) explained this discrepancy with the existence of small differences in specimen size and compaction procedure between these two sets of samples. With reference to the tests considered in this work, the average compaction void ratio of the two specimens tested in saturated conditions and shown in Figure 2 is 0.017 greater than the average compaction void ratio of the three specimens tested in unsaturated conditions and shown in Figure 1. To compensate for this difference, the experimental values of void ratio of the two saturated tests in Figure 2 have been reduced by 0.017 before calibrating the saturated normal compression line.

Note also that Sharma (1998) did not perform any saturated compression test but he presented a drying test under constant isotropic load which remained saturated throughout suction increase due to the high air entry value of the soil. The variation of void ratio against average skeleton stress over the virgin range of this drying test has therefore been used to calibrate the saturated normal compression line in Figure 2. 
By substituting Equation (4) into Equation (3), one obtains the following general expression that describes the normal compression behaviour of both saturated and unsaturated soils:

$$
\log e=-\lambda_{p} \log \frac{\bar{p}}{p_{r e f}^{\prime}}
$$

where $\bar{p}$ is an auxiliary stress variable defined as

$$
\bar{p}=S_{r}^{\frac{\lambda_{r}}{\lambda_{p}}} \cdot p^{\prime}
$$

Note that, in Equation (5), the reference value of the average skeleton stress $p_{r e f}^{\prime}$ in saturated conditions coincides with the reference value of the auxiliary stress $\bar{p}_{r e f}$. This is because, in saturated conditions, the average skeleton stress $p^{\prime}$ coincides with the auxiliary stress $\bar{p}$ as shown by substituting $S_{r}=1$ in Equation (6). Therefore Equation (5) can also be written as:

$$
\log e=-\lambda_{p} \log \frac{\bar{p}}{\overline{\bar{p}_{r e f}}}
$$

The auxiliary stress $\bar{p}$ of Equation (6) is here named the "scaled stress" because of its ability of taking into account the effect of capillarity on mechanical behaviour by scaling the average skeleton stress with a power function of degree of saturation. This is analogous to the water retention model of Gallipoli et al. (2015) where the "scaled suction" $\bar{s}$ was defined by factoring the soil suction with a power function of void ratio to take into account the effect of deformation on retention behavior. The scaled stress can therefore be regarded as the mechanical dual of the scaled suction in the hydraulic model of Gallipoli et al. (2015).

In summary, Equation (7) provides the expression of a unified normal compression line that is valid in both saturated and unsaturated conditions. The unified normal compression line has the same slope and intercept of the saturated normal compression line but is formulated in terms of scaled stresses instead of effective stresses. Therefore, with respect to saturated conditions, only one additional parameter $\lambda_{r}$ is needed (inside the expression of the scaled stress) to describe the normally consolidated behaviour of unsaturated soils. 
The unified normal compression lines of the three soils considered in this study are plotted in Figure 3, which confirms the normalizing effect of the scaled stress and the consequent regularization of saturated and unsaturated behaviour onto a unique virgin line.

\section{Bounding surface mechanical model}

The previous sections have presented a framework capable of describing the isotropic virgin behaviour of both saturated and unsaturated soils by means of a single normal compression line. This unified normal compression line has the same slope and intercept of the saturated line but is formulated in terms of a scaled stress that takes into account the mechanical effect of capillarity on virgin behaviour. In order to define a mechanical model for isotropic stress states, it is now necessary to extend this formulation beyond virgin loading to over-consolidated states.

This extension is performed by using a similar approach to that adopted in the hysteretic retention model of Gallipoli et al. (2015). In that model, a region of admissible states was defined in the $\log S_{r}-\log \bar{s}$ plane between the main drying and wetting curves. The retention behaviour over this region was then related to the distance of the soil state from these two main curves. In the same way, a region of over-consolidated states is here defined in the $\log e-\log \bar{p}$ plane between the unified normal compression line and the zero scaled stress axis (which coincides with the zero average skeleton stress axis if $S_{r} \neq 0$ ). Inside this overconsolidated region, the derivative of void ratio during an increase of scaled stress (referred here as "loading") depends on the distance of the current stress state from the unified normal compression line. The loading curve is then obtained by closed form integration of this derivative as explained next.

\section{Loading paths}

We assume that, for any loading path, the derivative in the $\log e-\log \bar{p}$ plane is equal to the slope of the unified normal compression line $\lambda_{p}$ reduced by a factor that depends on the distance of the current soil state from this line. This condition is mathematically expressed as: 


$$
\left(\frac{\mathrm{d} \log e}{\mathrm{~d} \log \bar{p}}\right)_{l}=-\lambda_{p}\left(\frac{\bar{p}}{\overline{p_{i}}}\right)^{\gamma}
$$

where $\left(\frac{\mathrm{d} \log e}{\mathrm{~d} \log \bar{p}}\right)_{l}$ is the derivative of the generic loading path (the subscript " $l$ " indicates loading) and $\left(\frac{\bar{p}}{\overline{p_{i}}}\right)^{\gamma}$ is the reducing factor of the slope of the unified normal compression line $\lambda_{p}$. The reducing factor is a power function with exponent $\gamma$, which is a material parameter, of the ratio between the current value of mean scaled stress $\bar{p}$ and the "image" value of mean scaled stress $\bar{p}_{i}$ calculated from the unified normal compression line at the current value of void ratio. The ratio $\frac{\bar{p}}{\bar{p}_{i}}$ is therefore always smaller than one and increases towards one as the soil state moves closer to the unified normal compression line. The slope of any loading path will therefore progressively increase towards the slope of the unified normal compression line, which also means that any loading path will asymptotically tend towards the unified normal compression line.

From Equation (7), the image value of the mean scaled stress is calculated as $\bar{p}_{i}=\frac{\bar{p}_{r e f}}{e^{\frac{1}{\lambda p}}}$ and Equation (8) can therefore be rewritten as:

$$
\left(\frac{\mathrm{d} \log e}{\mathrm{~d} \log \bar{p}}\right)_{l}=-\left(\frac{\bar{p} e^{\frac{1}{\lambda_{p}}}}{\bar{p}_{r e f}}\right)^{\gamma} \lambda_{p}
$$

Interestingly, Equation (9) can be integrated in a closed form to give:

$$
\log e=-\lambda_{p} \log \left(\left(\frac{\bar{p}}{\bar{p}_{\text {ref }}}\right)^{\gamma}+C_{l}\right)^{\frac{1}{\gamma}}
$$

where $C_{l}$ is a constant of integration calculated by imposing a suitable boundary condition as later explained. The validity of the above integration can be verified by deriving both sides of Equation (10), which gives back Equation (9) after noting that $\mathrm{d} e / e=\mathrm{d} \log e, \mathrm{~d} \bar{p} / \bar{p}=\mathrm{d} \log \bar{p} \quad$ and $\left(\frac{\bar{p}}{\bar{p}_{\text {ref }}}\right)^{\gamma}+C_{l}=$ $e^{-\frac{\gamma}{\lambda_{p}}}$ 
Importantly, Equation (10) provides a general expression that describes all isotropic loading paths. This expression is always valid regardless of the level of over-consolidation and regardless of whether the soil is saturated or unsaturated, each loading path being characterized by a different value of the integration constant $C_{l}$. For example, when $C_{l}=0$, Equation (10) reduces to the unified normal compression line of Equation (7). This means that the unified normal compression line is a particular case of loading curve corresponding to an integration constant of zero.

By taking the exponential base 10 of both sides, Equation (10) can be alternatively written in the following form without logarithms in the plane of void ratio and scaled stress $(e-\bar{p})$ :

$$
e=\left(\left(\frac{\bar{p}}{\bar{p}_{\text {ref }}}\right)^{\gamma}+C_{l}\right)^{-\frac{\lambda_{p}}{\gamma}}
$$

Or, by introducing the definition of the mean scaled stress, Equation (11) can be recast as a loading surface in the space of void ratio, mean average skeleton stress and degree of saturation $\left(e-p^{\prime}-S_{r}\right)$ :

$$
e=\left(\left(\frac{p^{\prime} S_{r}^{\frac{\lambda_{r}}{\lambda_{p}}}}{\bar{p}_{r e f}}\right)^{\gamma}+C_{l}\right)^{-\frac{\lambda_{p}}{\gamma}}
$$

Equations (10), (11) and (12) are alternative and equivalent descriptions of a generic loading path (i.e. a path characterized by an increase of scaled stress) and are valid in both saturated and unsaturated conditions.

As mentioned before, the constant of integration $C_{l}$ can be calculated from Equation (10) by imposing a boundary condition corresponding to a known soil state $\left\{e_{o}, \bar{p}_{o}\right\}$ like, for example, the initial soil state:

$$
C_{l}=e_{o}^{-\frac{\gamma}{\lambda_{p}}}-\left(\frac{\bar{p}_{o}}{\bar{p}_{r e f}}\right)^{\gamma}
$$

In order for a soil state to remain on a loading path, the value of scaled stress $\bar{p}$ must monotonically increase. If a reversal of scaled stress occurs, the soil state moves from a loading curve to an unloading curve, which is described by a different equation as explained in the next section. This is again similar to the 
retention model of Gallipoli et al. (2015) where a soil path follows a drying equation as long as the scaled suction increases but, if the scaled suction starts to reduce, the soil state switches to a wetting curve, which is described by a different equation.

\section{Unloading paths}

Unloading paths are defined as soil paths characterized by a decrease of scaled stress. Unlike loading, we assume that the derivative of the generic unloading path in the $\log e-\log \bar{p}$ plane is constant and equal to the slope of the swelling line $\kappa$. This condition is mathematically expressed as:

$$
\left(\frac{\mathrm{d} \log e}{\mathrm{~d} \log \bar{p}}\right)_{u}=-\kappa
$$

where $\left(\frac{\mathrm{d} \log e}{\mathrm{~d} \log \bar{p}}\right)_{u}$ is the derivative of the generic unloading path (the subscript " $u$ " indicates unloading). Note that, according to Equation (14), the derivative of the logarithm of void ratio is constant with respect to the logarithm of scaled stress but not with respect to the logarithm of average skeleton stress or suction. Equation (14) is easily integrated to give:

$$
\log e=-\kappa \log \bar{p}+\log C_{u}
$$

where $C_{u}$ is a constant of integration whose value is calculated by imposing a suitable boundary condition as explained later.

Equation (15) provides a general expression that describes all isotropic unloading paths regardless of whether the soil is saturated or unsaturated. Similar to the loading case, Equation (15) can be rewritten in two alternative equivalent forms. The first one does not involve logarithms and is represented as a curve in the plane of void ratio and scaled stress $(e-\bar{p})$ :

$$
e=\frac{C_{u}}{\bar{p}^{\kappa}}
$$


The second one is obtained by substituting the definition of mean scaled stress into Equation (16), which now becomes a surface in the space of void ratio, mean average skeleton stress and degree of saturation $\left(e-p^{\prime}-S_{r}\right):$

$$
e=\frac{C_{u}}{\left(p^{\prime} S_{r}^{\frac{\lambda_{r}}{\lambda_{p}}}\right)^{\kappa}}
$$

The constant of integration $C_{u}$ is calculated by imposing a boundary condition corresponding to a known soil state like, for example, the start of the unloading path. During a loading-unloading cycle, the start of the unloading path coincides with the reversal point of the cycle and must therefore be the same as the final point of the previous loading path. By imposing the boundary condition $\left\{e_{o}, \bar{p}_{o}\right\}$, the value of the constant of integration is calculated from Equation (15) as:

$$
C_{u}=e_{o} \bar{p}_{o}{ }^{\kappa}
$$

\section{Model calibration}

The three parameters describing the virgin behaviour of the soil $\left(\lambda_{r}, \lambda_{p}, \bar{p}_{r e f}\right)$ have been previously calibrated against normally consolidated data for the three soils considered in this work. The remaining two parameters $(\gamma, \kappa)$, describing the over-consolidated behaviour, are next calibrated by fitting the loading and unloading branches of a scaled stress cycle, respectively.

The constant of integration of the first branch of the cycle is calculated by imposing that the initial points of the predicted and experimental curves are the same. Instead, the constant of integration of the subsequent branch is calculated by imposing the continuity of predictions at the reversal point, i.e. by imposing that the initial point of the subsequent branch coincides with the final point of the previous branch.

According to the definition of scaled stress in Equation (6), a cycle of scaled stress can be obtained in different ways, i.e. by performing a net stress cycle (loading-unloading), a suction cycle (drying-wetting) or a combination of the two. For the data by Raveendiraraj (2009), we have chosen a loading-unloading cycle 
at a constant suction of $150 \mathrm{kPa}$ where the mean net stress is increased from $20 \mathrm{kPa}$ to $150 \mathrm{kPa}$ and decreased back to $75 \mathrm{kPa}$ (see Figure 4a). Instead, for the data by Sharma (1998), we have chosen a wetting-drying cycle at a constant mean net stress of $10 \mathrm{kPa}$ between suctions of $20 \mathrm{kPa}$ and $300 \mathrm{kPa}$ (see Figure 4b). For the data by Sivakumar (1993), no cycle of net stress or suction is available, thus only the parameter $\gamma$ has been calibrated by best-fitting the loading path of an over-consolidated soil subjected to an increase of mean net stress from $50 \mathrm{kPa}$ to $300 \mathrm{kPa}$ at a constant suction of $200 \mathrm{kPa}$ (see Figure 4c).

During the above simulations, the value of the scaled stress has been calculated according to Equation (6) by using the experimental values of degree of saturation. This value of scaled stress has then been introduced in Equation (10) or (15), depending on whether a loading path or an unloading path is simulated. The use of experimental values of degree of saturation inside the scaled stress equation is here acceptable because the present model predicts the volumetric behaviour of the soil and not the retention behaviour. Nevertheless, for practical applications, a suitable retention law will need to be coupled with the present formulation to calculate the degree of saturation during a generic stress/suction path.

In Figure 4, the good fit of the calibrated curves to experimental data indicates that the chosen parameter values are capable of correctly predicting the soil behaviour along different types of stress paths. In the case of Sharma (1998) (Figure 4b), the small discrepancy between experimental and predicted behaviour towards the end of the suction cycle is probably due to the fast drying rate adopted in this test, which impeded equalization of suction inside the sample (see Sharma, 1998).

Because of the definition of scaled stress given by Equation (6), the reversal points of a scaled stress cycle may in principle be different from the reversal point of the corresponding net stress or suction cycle. It is therefore recommendable that, during calibration, experimental data are plotted in terms of scaled stress to ensure that reversal points are correctly identified and the increasing and decreasing branches of the scaled stress cycle are properly separated.

The values of all five model parameters $\left(\lambda_{r}, \lambda_{p}, \bar{p}_{r e f}, \gamma, \kappa\right)$ for the three soils considered in this work are summarized in Table 1. With respect to the saturated case, only one additional parameter $\lambda_{r}$ is needed to describe the unsaturated virgin behaviour of the soil. Also, only one extra parameter $\gamma$ is needed to 
incorporate a smooth transition from over-consolidated to normally consolidated states for both saturated and unsaturated soils.

As an alternative calibration procedure, all five parameters $\left(\lambda_{r}, \lambda_{p}, \bar{p}_{r e f}, \gamma, \kappa\right)$ could have been simultaneously determined by fitting one or more cycles of scaled stress, instead of separately calibrating the three virgin parameters $\left(\lambda_{r}, \lambda_{p}, \bar{p}_{r e f}\right)$ against normally consolidated data as done above. If such alternative calibration approach is followed, it is recommended that different types of cycles, e.g. a net stress cycle and a suction cycle, are simultaneously fitted to enhance the robustness of calibration. This alternative calibration approach has the advantage of being faster but has also the drawback of hiding the physical meaning of model parameters and possibly requiring specific software for the multi-variable fitting of experimental data.

\section{Model validation}

The model response has been validated against experimental data not used during the previous calibration. To make the validation more testing, the types of stress paths chosen during validation differ from those used during calibration. This means that, if a mean net stress cycle has been employed to calibrate the model, a suction cycle has instead been used during validation. Vice versa, if the model has been calibrated against a suction cycle, a mean net stress cycle has been employed during validation.

For the data by Raveendiraraj (2009), the model has been validated against a drying-wetting-drying cycle at a constant mean net stress of $10 \mathrm{kPa}$ where suction was increased from $30 \mathrm{kPa}$ to $300 \mathrm{kPa}$, then reduced to $40 \mathrm{kPa}$ and finally increased again to $200 \mathrm{kPa}$. At the end of this suction cycle, the soil was isotropically loaded from a mean net stress of $10 \mathrm{kPa}$ to a mean net stress of $275 \mathrm{kPa}$. This final loading was performed at a constant suction of $200 \mathrm{kPa}$, which differs from the suctions levels of $50 \mathrm{kPa}, 150, \mathrm{kPa}$ and $350 \mathrm{kPa}$ considered during calibration. A good agreement exists between predicted and experimental data during both the initial suction cycle (Figure 5a) and the subsequent loading path (Figure 5b).

The ability of the model to capture wetting-induced collapse compression has also been validated against a different test by Raveendiraraj (2009) in which suction was gradually reduced from $300 \mathrm{KPa}$ to $100 \mathrm{kPa}$ at a 
constant mean net stress of $101 \mathrm{kPa}$ and a constant deviatoric stress of $78 \mathrm{kPa}$. The present model is restricted to isotropic stress states and therefore the application of a constant deviatoric stress has been neglected during the simulation. Yet, Figure $5 \mathrm{c}$ shows a good agreement between predicted and experimental data indicating that the magnitude of collapse compression is captured with reasonable accuracy by the model.

The proposed model can therefore predict both wetting-induced swelling (path BC in Figure 5a) and wetting-induced collapse compression (path $A B$ in Figure $5 c$ ) by using a single stress variable. This is possible because the mean scaled stress expression of Equation (6) is the product of two terms, i.e. the capillary bonding factor $S_{r}^{\frac{\lambda_{r}}{\lambda_{p}}}$ and the mean average skeleton stress $p^{\prime}=p^{\text {net }}+S_{r} s$, which evolve in opposite directions during wetting at constant net stress because saturation rises and suction reduces. Therefore, the mean scaled stress can either increase (inducing collapse compression) or decrease (inducing swelling) depending on which of these two terms dominates.

Consider, for example, a soil element subjected to a wetting path at constant mean net stress $p^{\text {net }}$ from an initial unsaturated state $\left(S_{r}<1 ; s>0\right)$ to a final saturated state $\left(S_{r}=1 ; s=0\right)$. During this path, the scaling factor increases from $S_{r}^{\frac{\lambda_{r}}{\lambda_{p}}}$ to one while the mean average skeleton stress reduces from $p^{\text {net }}+S_{r} S$ to $p^{n e t}$, which results in a variation of mean scaled stress from $S_{r}^{\frac{\lambda_{r}}{\lambda_{p}}}\left(p^{n e t}+S_{r} s\right)$ to $p^{n e t}$. The change of mean scaled stress $\Delta \bar{p}$ can therefore be calculated as

$$
\Delta \bar{p}=p^{n e t}-S_{r}^{\frac{\lambda_{r}}{\lambda_{p}}}\left(p^{n e t}+S_{r} s\right)=\left(1-S_{r}^{\frac{\lambda_{r}}{\lambda_{p}}}\right) p^{n e t}-S_{r}^{\frac{\lambda_{r}}{\lambda_{p}}+1} s
$$

and can be either positive (producing collapse compression) or negative (producing swelling) depending on whether the load term $\left(1-S_{r}^{\frac{\lambda_{r}}{\lambda_{p}}}\right) p^{\text {net }}$ is bigger or smaller than the suction term $S_{r}^{\frac{\lambda_{r}}{\lambda_{p}}+1} S$. In the case of Sharma (1998), the model has been validated against a double loading-unloading cycle at a constant suction of $200 \mathrm{kPa}$. During this cycle, the soil was first loaded from an initial mean net stress of 10 
$\mathrm{kPa}$ to a mean net stress of $100 \mathrm{kPa}$, then unloaded back to $10 \mathrm{kPa}$ and subsequently reloaded to $250 \mathrm{kPa}$ before being finally unloaded to $110 \mathrm{kPa}$. The results of this simulation are shown in Figure 6 , which indicates again an excellent agreement between predicted and experimental values.

Finally, in the case of Sivakumar (1993), no cycle of mean net stress or suction is available. Therefore, the model has been validated against a simple loading path of an over-consolidated soil subjected to an increase of mean net stress from $50 \mathrm{kPa}$ to $150 \mathrm{kPa}$ at a constant suction of $300 \mathrm{kPa}$. The agreement between experimental data and model predictions is again very good (Figure 7).

Sivakumar (1993) also measured the wetting induced collapse compression of a number of samples saturated, shortly after compaction, by flushing water through them under a small isotropic stress. Table 2 summarizes the average experimental values of degree of saturation, pore pressure, stress and void ratio of these samples in the as-compacted state and after saturation (Sivakumar and Wheeler, 2000; Wheeler and Sivakumar, 1995). Table 2 also presents the values of void ratio predicted by the proposed model assuming monotonic loading from the initial as-compacted state to the final saturated state with an integration constant $C_{l}$ obtained by imposing the coincidence of the predicted and experimental values of void ratio in the initial as-compacted state. The assumption of loading is justified by the observed increase of scaled stress from the initial to the final state. The monotonicity of loading is instead a simplifying assumption which has been introduced in the absence of any information about the actual stress path followed by the soil during flushing. Inspection of Table 2 indicates that, despite this simplifying assumption, the model is capable of capturing the occurrence of collapse compression during saturation by predicting a reduction of void ratio which is however slightly smaller than that observed experimentally.

In all the above simulations, the first integration constant has been calculated by imposing the coincidence of the predicted and experimental curves at the start of the test. Moreover, in the presence of a reversal of scaled stress, the integration constant has been updated by imposing the continuity of the predicted curve at the reversal point, i.e. by imposing that the first point after reversal coincides with the last point before reversal. Similar to calibration, experimental values of degree of saturation have been used to calculate the 
scaled stress in all simulations, which is the reason why some of the predicted curves appear slightly jagged (see, for example, Figure 7).

\section{Conclusions}

This paper has demonstrated the existence of a unified normal compression line for both saturated and unsaturated soils, whose definition stems from consideration of the capillary bonding between soil grains. This unified normal compression line has the same slope and intercept of the saturated normal compression line but is formulated in terms of an alternative stress variable that accounts for the mechanical effect of capillarity. This variable has been named the "scaled stress" because is equal to the average skeleton stress scaled by a power function of degree of saturation and reduces to Terzaghi's effective stress under saturated conditions. The effect of suction and/or degree of saturation on mechanical behaviour is therefore incorporated in the definition of the scaled stress, which means that an explicit dependency of the normal compression line on these two variables is no longer necessary. The scaled stress can be regarded as the mechanical dual of the scaled suction in the retention model of Gallipoli et al. (2015), where the effect of deformation on water retention was similarly introduced by scaling soil suction with a power function of void ratio.

Following upon the definition of the unified normal compression line, the paper has also formulated a bounding surface model for over-consolidated soils subjected to isotropic loading or unloading. In this model, no classical distinction exists between elastic and elasto-plastic behaviour but irreversible deformations occur gradually from the very beginning of a loading or unloading path.

During loading, gradual yielding is predicted by assuming that the slope of the generic loading curve increases towards the slope of the unified normal compression line as the soil state approaches this line. The slope of the generic loading curve is then integrated to provide a general closed-form expression that is valid for all loading paths in both saturated and unsaturated conditions. Different loading paths are distinguished by different values of the integration constants, which are calculated by imposing suitable boundary conditions. This represents another similarity with the retention model of Gallipoli et al. (2015) 
where drying and wetting paths are described by two general expressions whose slope varies with the distance of the soil state from the corresponding main curve.

The proposed model uses a total of five parameters, which include the three standard parameters describing the elasto-plastic behaviour of saturated soils under isotropic stress states (i.e. the slope and intercept of the saturated normal compression line and the slope of the swelling line) plus two extra parameters that govern the unsaturated behaviour and the gradual yielding of the soil, respectively. All five parameters have a clear physical interpretation which facilitates their calibration from laboratory tests. In this work, parameter values have been selected by fitting laboratory data over a relatively large range of saturations from 0.6 to 1 . Over this interval, the parameter $\lambda_{r}$ controlling the unsaturated behaviour varies relatively little from 0.490 , for a compacted kaolin, to 0.521 , for a compacted kaolin/bentonite mix.

Although the proposed model is limited to isotropic stress states, it provides the basis for the formulation of a full constitutive framework to describe the mechanical behaviour of both saturated and unsaturated soils under general stress states by using a single stress variable.

\section{REFERENCES}

Alonso EE, Gens A and Josa A (1990). A constitutive model for partially saturated soils. Géotechnique 40(3): 405-430.

Bolzon G, Schrefler BA and Zienkiewicz OC (1996). Elastoplastic soil constitutive laws generalised to partially saturated states. Géotechnique 46(2): 279-289, http://dx.doi.org/10.1680/geot.1996.46.2.279

Butterfield R (1979). A natural compression law for soils (an advance on e - log p'). Géotechnique 29(4): 469 $-480$.

Cui YJ and Delage P (1996). Yielding and plastic behaviour of an unsaturated compacted silt. Géotechnique 46: 291-311. 
Fisher RA (1926). On the capillary forces in an ideal soil correction of formulae given by WB Haines. Journal Agricultural Science 16(3): 492-505.

Gallipoli D, Bruno AW, D'Onza F and Mancuso C (2015). A bounding surface hysteretic water retention model for deformable soils. Géotechnique 65(10): 793-804, http://dx.doi.org/10.1680/igeot.14.P.118

Gallipoli D, Gens A, Sharma R and Vaunat J (2003). An elasto-plastic model for unsaturated soil incorporating the effects of suction and degree of saturation on mechanical behaviour. Géotechnique $\mathbf{5 3}$ (1): 123-135, http://dx.doi.org/10.1680/geot.2003.53.1.123

Hu R, Liu HH, Chen Y, Zhou C and Gallipoli D (2014). A constitutive model for unsaturated soils with consideration of inter-particle bonding. Computers and Geotechnics 59: 127-144

Jommi C and Di Prisco C (1994). Un semplice approccio teorico per la modellazione del comportamento meccanico dei terreni granulari parzialmente saturi (in Italian). In Atti Convegno sul Tema: II Ruolo deiFluidi nei Problemi di Ingegneria Geotecnica, Mondoví, pp. 167-188.

Khalili N, Habte MA and Zargarbashi S (2008). A fully coupled flow deformation model for cyclic analysis of unsaturated soils including hydraulic and mechanical hysteresis. Computers and Geotechnics 35(6): 872889.

Lourenço SDN, Gallipoli D, Augarde CE, Toll DG, Fisher PC and Congreve A (2012). Formation and evolution of water menisci in unsaturated granular media. Géotechnique 62(3): 193-199, http://dx.doi.org/10.1680/geot.11.P.034

Manzanal D, Pastor M and Fernandez Merodo JA (2011). Generalized plasticity state parameter-based model for saturated and unsaturated soils. Part II: Unsaturated soil modeling. International Journal for Numerical and Analytical Methods in Geomechanics 35(18): 1899-1917.

Mašín D and Khalili N (2008). A hypoplastic model for mechanical response of unsaturated soils. International Journal for Numerical and Analytical Methods in Geomechanics 32(15): 1903-1926. 
McDowell GR (2005). A physical justification for log e-log $\sigma$ based on fractal crushing and particle kinematics. Géotechnique 55(9):697-698.

Nuth M and Laloui L (2007). New insight into the unified hydromechanical constitutive modelling of unsaturated soils. Proc. 3rd Asian Conference on Unsaturated Soils (Yin Z.Z., Yuan Y.P. and Chiu A.C.F. eds.). Science Press, Beijing, pp. 109-126.

Pereira JM, Wong H, Dubujet $P$ and Dangla $P$ (2005). Adaptation of existing behaviour models to unsaturated states: application to CJS model. International Journal for Numerical and Analytical Methods in Geomechanics 29(11): 1127-1155.

Pestana JM and Whittle AJ (1995). Compression model for cohesionless soils. Géotechnique 45(4):611-631.

Raveendiraraj A (2009). Coupling of mechanical behaviour and water retention behaviour in unsaturated soils, PhD thesis, University of Glasgow, UK.

Sharma RS (1998). Mechanical behaviour of unsaturated highly expansive clays, PhD thesis, University of Oxford, UK.

Sheng D, Fredlund DG and Gens A (2008). A new modelling approach for unsaturated soils using independent stress variables. Canadian Geotechnical Journal 45(4): 511-534.

Sivakumar V (1993). A critical state framework for unsaturated soil, PhD thesis, University of Sheffield, UK.

Sivakumar V and Wheeler SJ (2000). Influence of compaction procedure on the mechanical behaviour of an unsaturated compacted clay; Part 1: Wetting and isotropic compression. Géotechnique 50(4): 359-368.

Tarantino A and Tombolato S (2005). Coupling of hydraulic and mechanical behaviour in unsaturated compacted clay. Géotechnique 55(4): 307-317.

Toll DG and Ong BH (2003). Critical-state parameters for an unsaturated residual sandy clay. Geotechnique 53(1): 93-103. 
Vaunat J, Romero E and Jommi C (2000). An elastoplastic hydro-mechanical model for unsaturated soils. Experimental evidence and theoretical approaches in unsaturated soils (Eds. Tarantino A. and Mancuso C.), 121-138. Rotterdam: Balkema.

Wheeler SJ, Sharma RS and Buisson MSR (2003). Coupling of hydraulic hysteresis and stress-strain behaviour in unsaturated soils. Géotechnique 53(1): 41-54.

Wheeler SJ and Sivakumar V (1995). An elastoplastic critical state framework for unsaturated soil. Géotechnique 45(1): 35-53.

Zhou C, Ng CWW and Chen R (2015). A bounding surface plasticity model for unsaturated soil at small strains. International Journal for Numerical and Analytical Methods in Geomechanics 39(11): 1141-1164 
TABLE AND RELATIVE CAPTION

Table 1. Model parameter values

\begin{tabular}{cccccc}
\hline & $\lambda_{r}$ & $\lambda_{p}$ & $\bar{p}_{\text {ref }}$ & $\gamma$ & $\kappa$ \\
\hline $\begin{array}{c}\text { Raveendiraraj (2009) } \\
\text { compacted kaolin }\end{array}$ & 0.519 & 0.124 & $83 \mathrm{kPa}$ & 4.00 & 0.039 \\
$\begin{array}{c}\text { Sharma (1998) } \\
\text { compacted bentonite/kaolin mix }\end{array}$ & 0.521 & 0.160 & $200 \mathrm{kPa}$ & 5.42 & 0.061 \\
$\begin{array}{c}\text { Sivakumar (1993) } \\
\text { compacted kaolin }\end{array}$ & 0.490 & 0.125 & $164 \mathrm{kPa}$ & 7.15 & $\begin{array}{c}\text { Not } \\
\text { applicable }\end{array}$ \\
\hline
\end{tabular}

Table 2. Prediction of saturation induced collapse compression (Sivakumar, 1993)

\begin{tabular}{|c|c|c|c|c|c|c|c|c|c|}
\hline & \multicolumn{7}{|c|}{ Average experimental values } & \multicolumn{2}{|c|}{ Predicted values } \\
\hline & $\begin{array}{l}S_{r} \\
(-)\end{array}$ & $\begin{array}{c}u_{a} \\
(\mathrm{kPa})\end{array}$ & $\begin{array}{c}u_{w} \\
(\mathrm{kPa})\end{array}$ & $\begin{array}{c}p^{t o t} \\
(\mathrm{kPa})\end{array}$ & $\begin{array}{c}p^{\prime} \\
(\mathrm{kPa})\end{array}$ & $\begin{array}{c}\bar{p} \\
(\mathrm{kPa})\end{array}$ & $\begin{array}{c}e \\
(-)\end{array}$ & $\begin{array}{c}e \\
(-)\end{array}$ & $\begin{array}{l}C_{l} \\
(-)\end{array}$ \\
\hline $\begin{array}{c}\text { Initial } \\
\text { as-compacted } \\
\text { state }\end{array}$ & 0.54 & 0 & -700 & 0 & 378 & 33.8 & 1.21 & 1.21 & \\
\hline $\begin{array}{l}\text { Final } \\
\text { saturated } \\
\text { state }\end{array}$ & 1 & No air & 35 & 75 & 40 & 40 & 1.16 & 1.19 & $5.9 \times 10^{-6}$ \\
\hline
\end{tabular}




\section{FIGURES AND RELATIVE CAPTIONS}
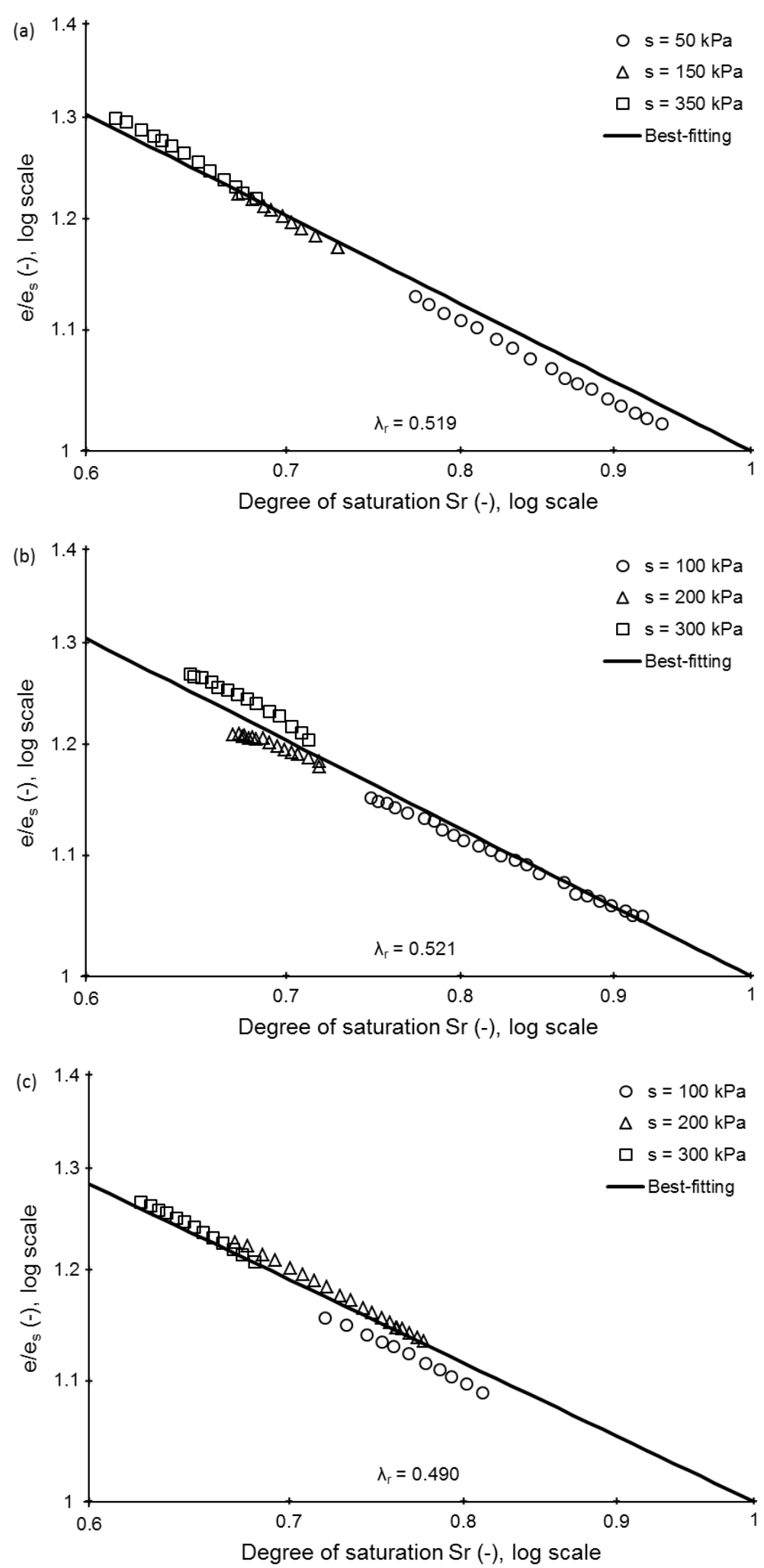

Figure 1. Fitting of proposed capillary bonding function to experimental data by: (a) Raveendiraraj (2009), (b) Sharma (1998) and (c) Sivakumar (1993). 

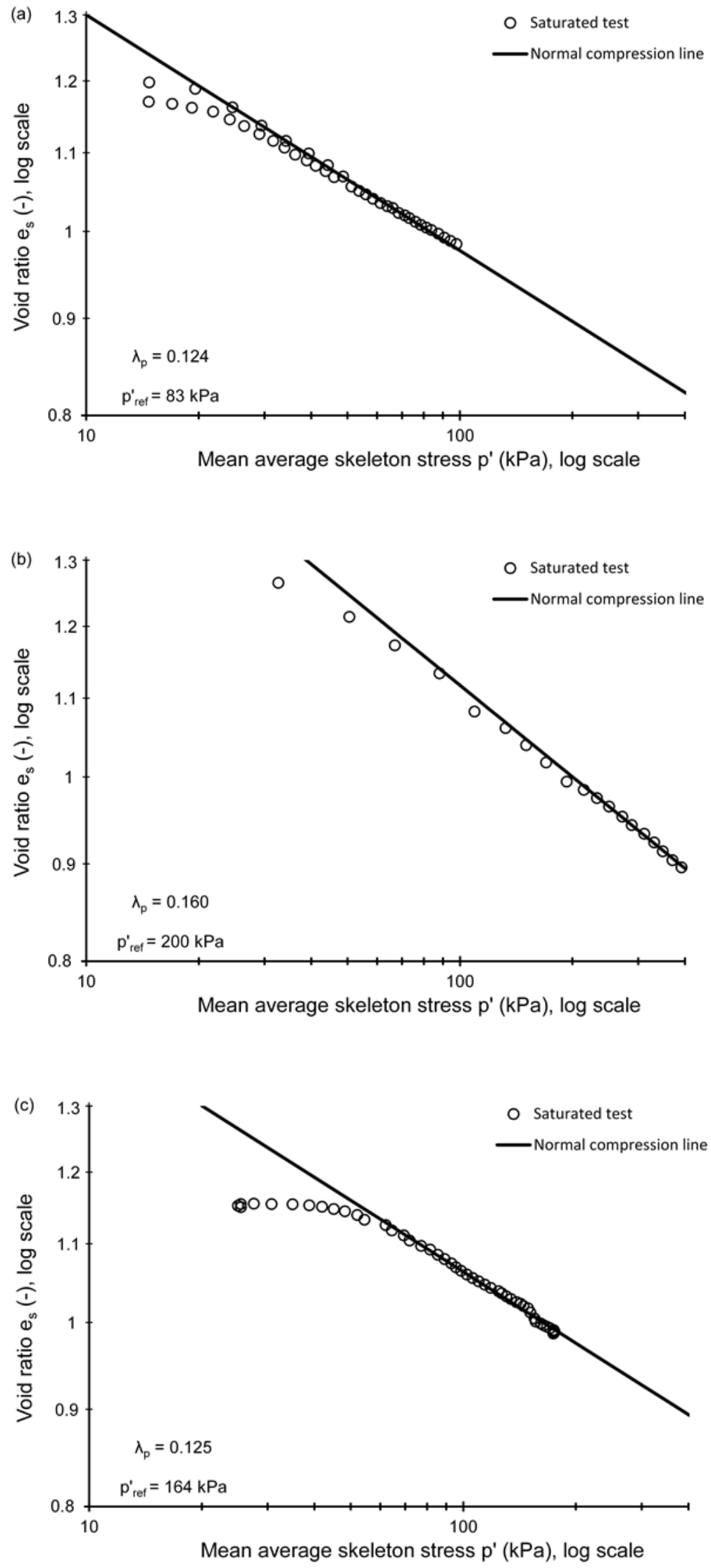

Figure 2. Fitting of double-logarithmic saturated normal compression line to experimental data by: (a) Raveendiraraj (2009), (b) Sharma (1998) and (c) Sivakumar (1993). 

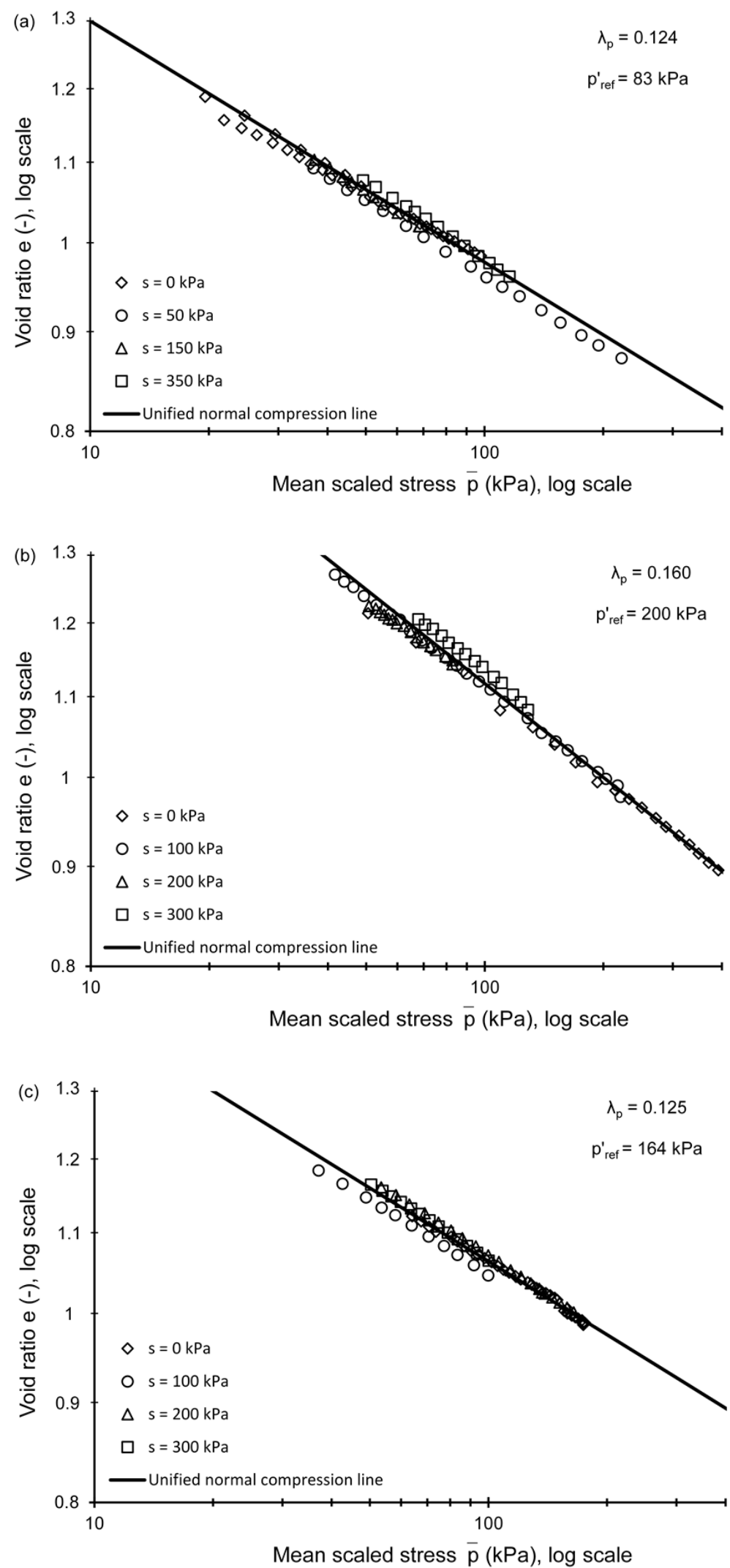

Figure 3. Predicted unified normal compression line against experimental data by: (a) Raveendiraraj (2009), (b) Sharma (1998) and (c) Sivakumar (1993). 

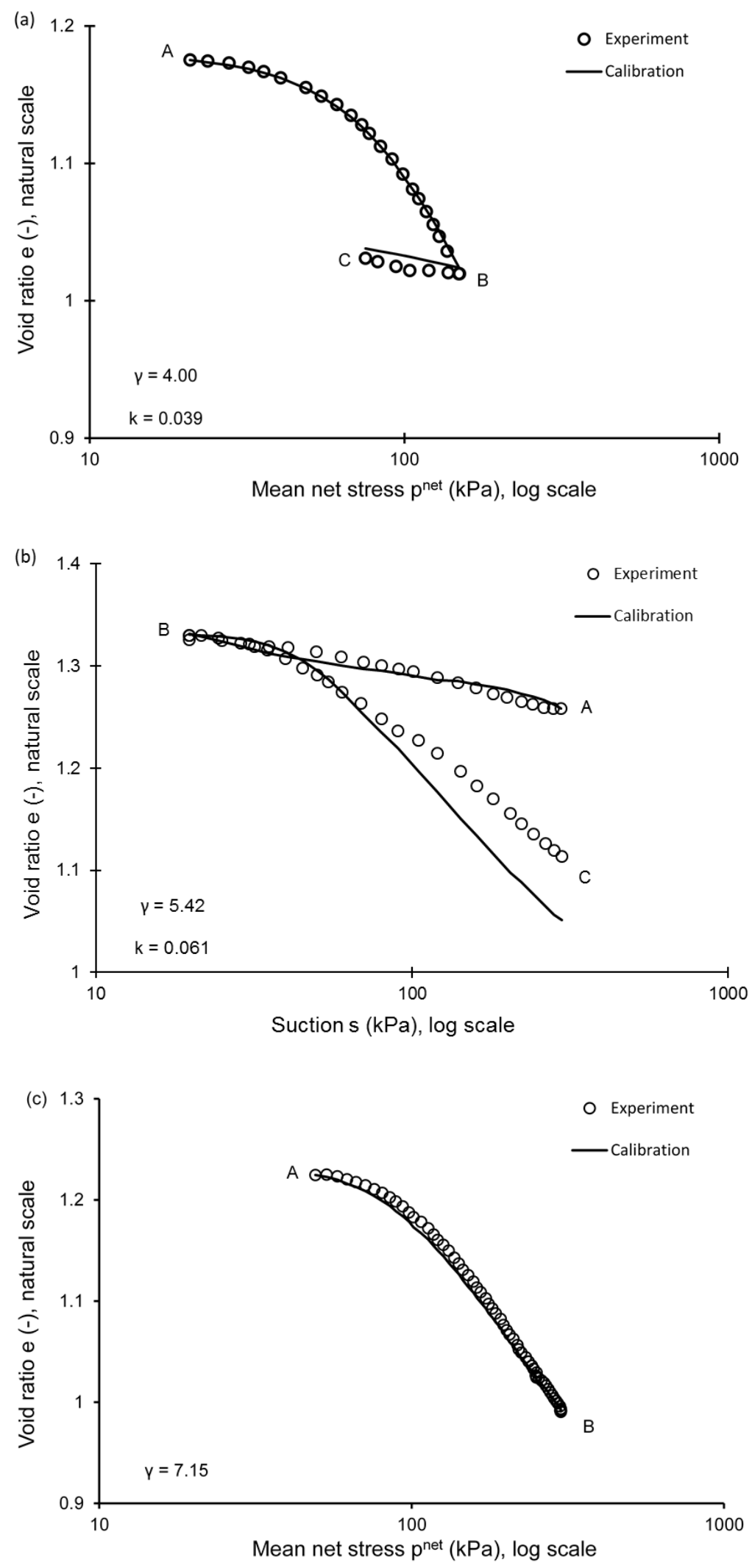

Figure 4. Calibration of parameters $\gamma$ and $\kappa$ against experimental data by: (a) Raveendiraraj (2009), (b) Sharma (1998) and (c) Sivakumar (1993). 

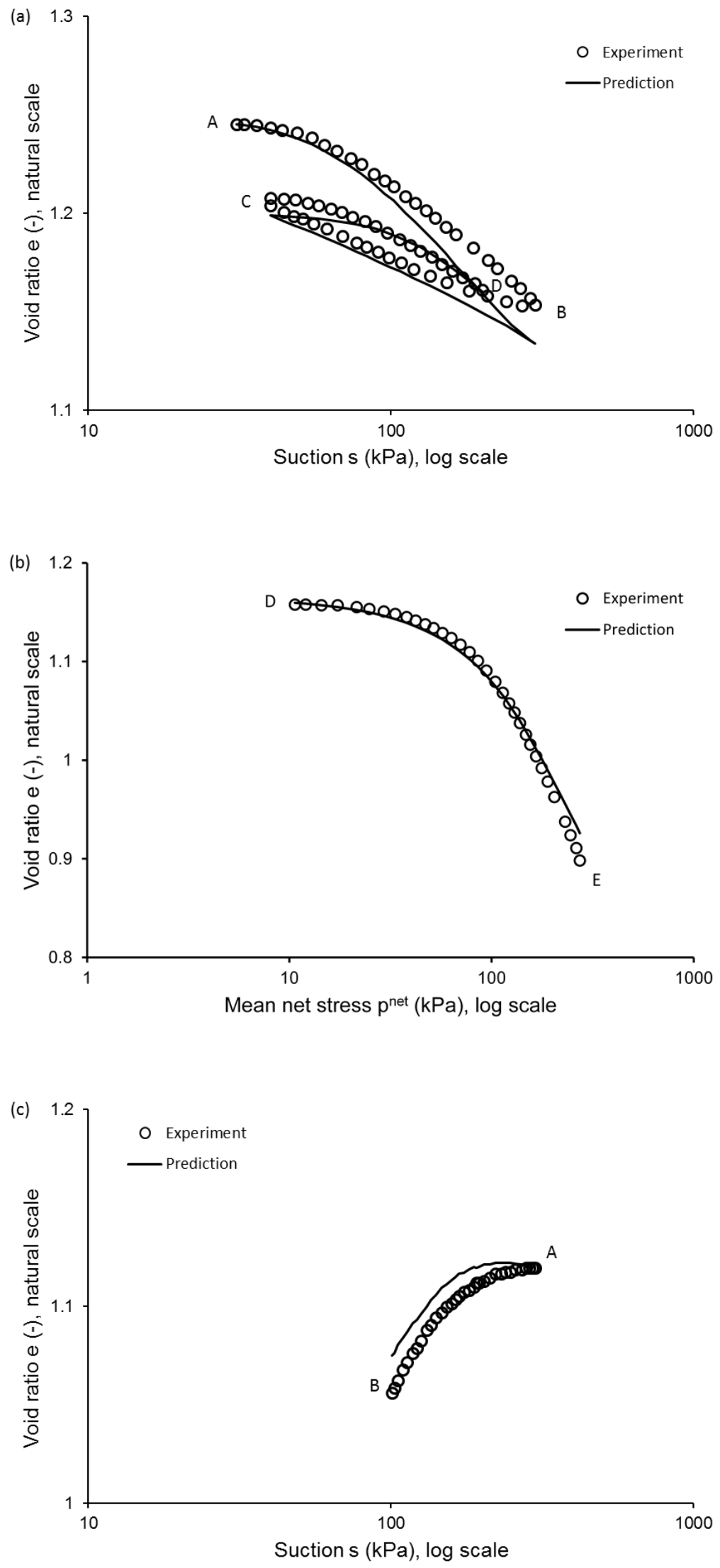

Figure 5. Validation of the proposed bounding surface model against experimental data by Raveendiraraj (2009): (a) suction cycle, (b) net stress loading and (c) wetting induced collapse compression. 


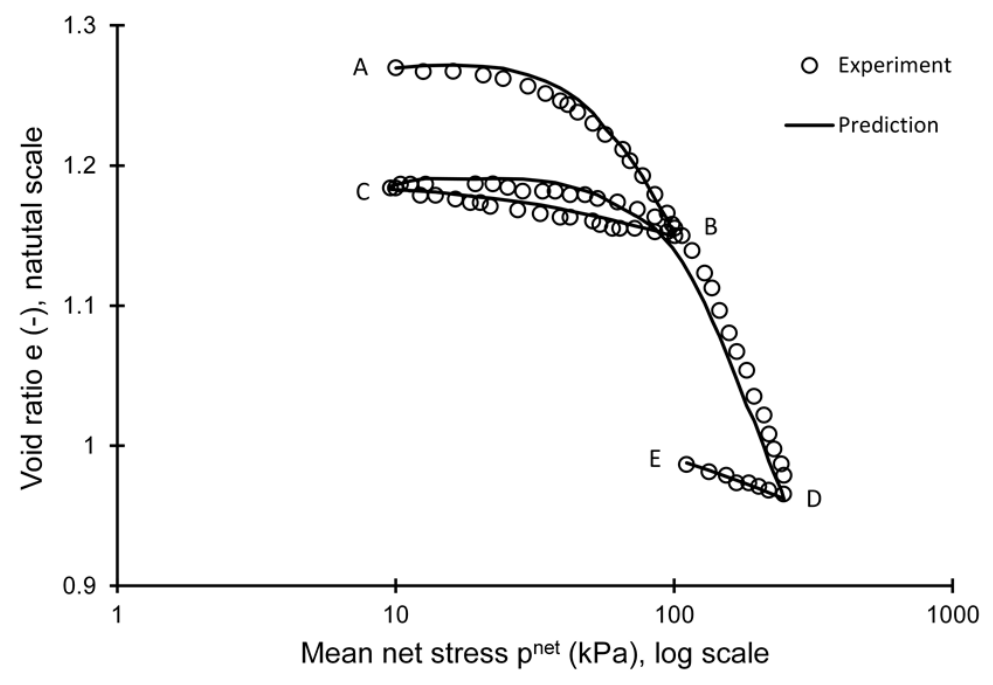

Figure 6. Validation of the proposed bounding surface model against experimental data by Sharma (1998): net stress cycle.

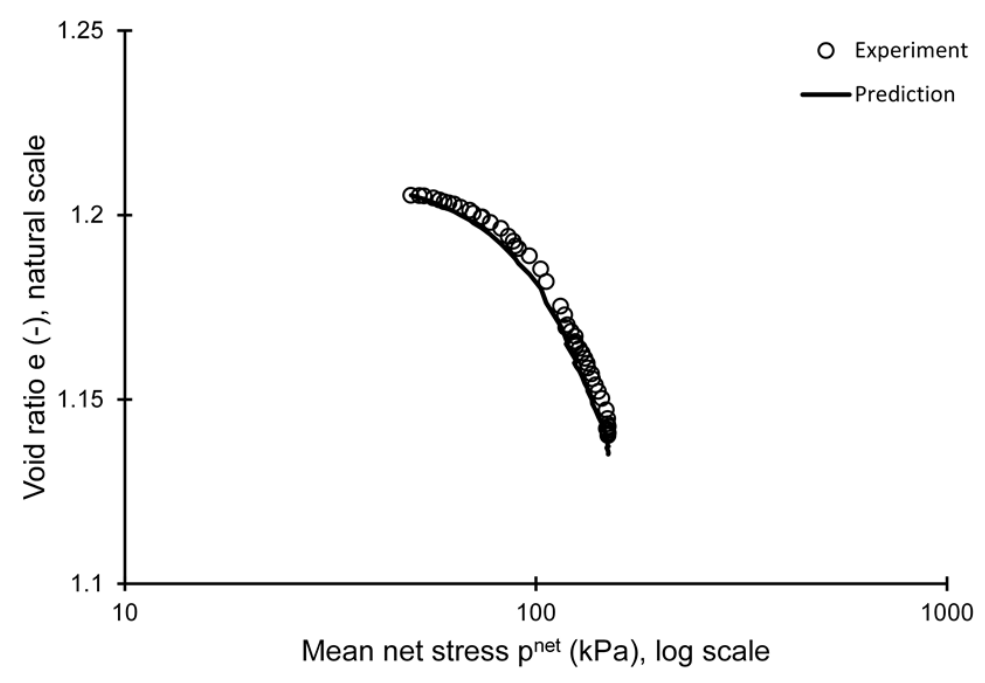

Figure 7. Validation of the proposed bounding surface model against experimental data by Sivakumar (1993): net stress loading. 